\title{
The effects of star spots on transit photometry
}

\author{
John R. Hodgson II and Damian J. Christian
}

Physics and Astronomy Department, California State University, Northridge 18111 Nordhoff Street, Northridge, California 91330-8268, United States of America email: John.Hodgson.71@my.csun.edu

Damian.Christian@csun.edu

\begin{abstract}
We have undertaken an observational program to photometrically monitor several transiting planet host stars. The Rabus et al. result for TrES-1 showed the dramatic effects star spots can have on transit photometry. We will investigate the effects of spots on transit light curves and estimates of planetary radii. The observed spot patterns will be used to derive the rotational periods of our sample. Our sample includes several of the newly discovered transiting ESPs from the SuperWASP, HAT, TrES, and Kepler projects.
\end{abstract}

Keywords. Stars: starspots, planetary systems, techniques: photometric

\section{Introduction}

Currently, the discovery of new extra-solar planets is occurring nearly every day. Approximately $20 \%$ of these are 'transiting' extra-solar planets (TESPs) found through photometric observations of their host stars. Precision photometry combined with radial velocity measurements constrain the values of the planetary radii in addition to the calculated inclination angle of the planetary system. Variations in the photometry of a planetary system is often due to stellar activity. While these variations are normally detrimental to establishing the physical parameters of the planetary system, they can be used to gain information about the host stars.

Stellar activity is a prominent problem in deducing the planetary parameters of late type stars. The occultation of stellar activity by a planet changes the shape of the transit light curve and can lead to incorrect estimates of the planet's radius. An example of this occurred during observations of the active K0V star TrES-1 and was reported by (Rabus et al. 2009, Rabus et al. 2009). Their report stimulated a search for other instances of stellar activity on Tres-1 and other stars with known transiting extra-solar planets. Additionally, planetary transits may be used to infer physical properties of stellar spots, and the multiple transits easily available from space-based missions, such as CoRoT and Kepler have been used for these studies (Silva-Valio et al. 2010).

\section{Overview}

We have undertaken an observational program to photometrically monitor several transiting planet late-type host stars that are expected to have starspots. We are interested in investigating how starspots effect the transit depth and derived planetary radii. Additionally, information on stellar rotation rates can be determined from stellar activity present over multiple transits. In the current paper, we present our initial findings for this project. 
Table 1. Known characteristics of targets selected for follow-up photometry.

\begin{tabular}{|c|c|c|c|c|c|c|c|}
\hline $\begin{array}{l}\text { Target } \\
\text { Name }\end{array}$ & $\begin{array}{c}\text { Spectral } \\
\text { Type }\end{array}$ & $\begin{array}{c}\text { Stellar } \\
\text { Magnitude } \\
{[\text { V] }}\end{array}$ & $\begin{array}{l}\text { Stellar } \\
\text { Radius } \\
{\left[R_{S \text { un }}\right]}\end{array}$ & $\begin{array}{c}\text { Rotational } \\
\text { Period } \\
\text { [days] }\end{array}$ & $\begin{array}{c}\text { Planet } \\
\text { Radius } \\
{\left[R_{J}\right]}\end{array}$ & $\begin{array}{l}\text { Planetary } \\
\text { Period } \\
\text { [days] }\end{array}$ & $\begin{array}{l}\text { Tnclination } \\
\text { [degrees] }\end{array}$ \\
\hline WASP-10 & K5 & 12.7 & 0.783 & $11.9 \pm 0.2$ & 1.08 & 3.093 & 86.8 \\
\hline Kepler-6 & G1 & 13 & 1.391 & $\approx 6$ & 1.323 & 3.234 & 86.8 \\
\hline Kepler-7 & $\mathrm{F} 8 \backslash \mathrm{G} 0$ & 13.9 & 1.843 & & 1.478 & 4.886 & 86.5 \\
\hline Kepler-8 & $\mathrm{F} 7 \backslash 9$ & 13.9 & 1.486 & & 1.419 & 3.523 & 84.07 \\
\hline TrES-1 & $\mathrm{K} 0 \mathrm{~V}$ & 11.79 & 0.82 & $40.2_{-14.6}^{+22.9}$ & 1.081 & 3.030 & 86.4 \\
\hline
\end{tabular}

Table 1 contains an overview of some of our primary targets that have been selected for follow-up photometry.

Below is a list of the properties we will be looking for.

Planetary Radii. The radii of exoplanets is found from the change in the host star's intensity during a transit. When a starspot is occulted by the transiting planet, a decrease in the transit depth results which can cause an underestimate of the planet's radius. Similarly, when a plage is occulted by a transiting planet, an increase in the transit depth results which can cause an increase in the calculated planetary radii.
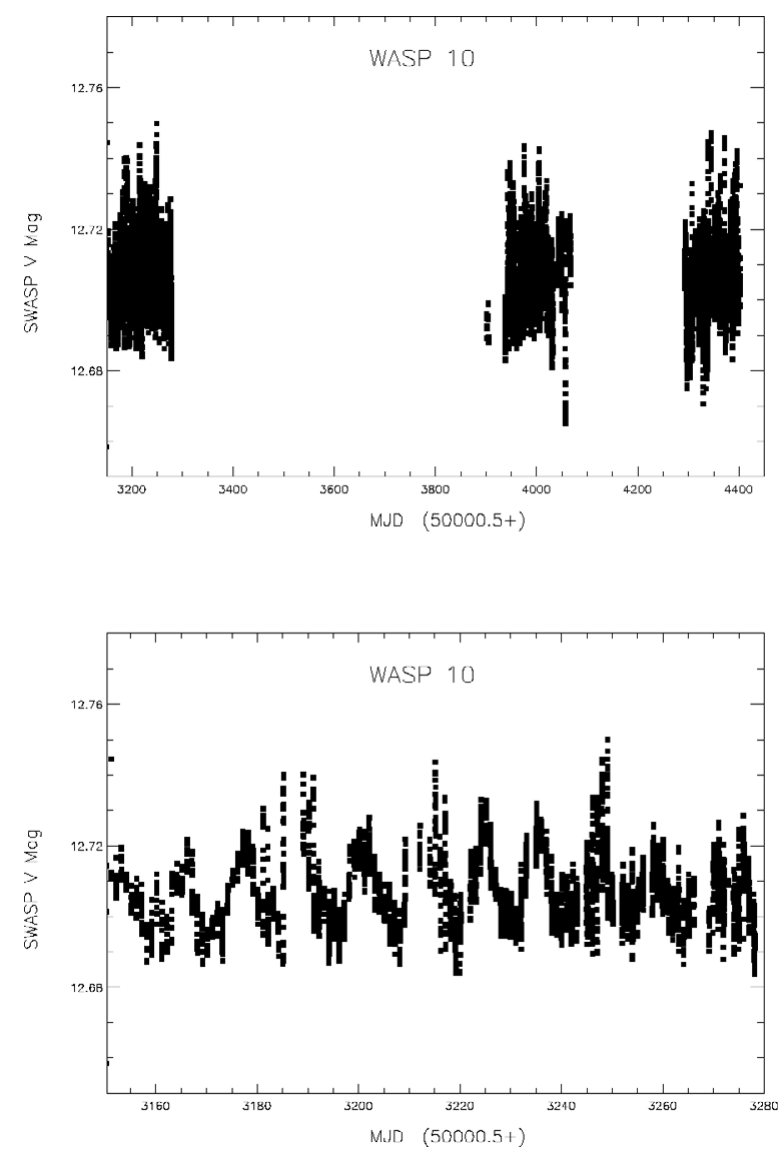

Figure 1. SuperWasp lightcurve of WASP-10 data from 2004 to 2007 (1 January 2004 is JD 2453005.5). The lower image is an expanded view of the smoothed 2004 WASP-10 data showing the 11.9 day period. 
Stellar Rotation. The observed occultations of a star spot over multiple transits can yield the rotation rate of the host star. Given the radius of the star, one only needs to measure the distance the spot has migrated across the stellar disk and the time between the transit measurements (Silva-Valio 2008). Assuming no change in the spot's area, variations in the modulations caused by the spot can be used to measure the inclination of the stellar rotational axis.

\section{Discussion}

In the first phase of our program we have analyzed photometric data from the SuperWASP and Kepler archives and searched for possible stellar rotation periods. We have focused on the stars in Table 1. We have determined a rotational period for WASP-10 of 11.9 days (see Figure 1) in agreement with Christian et al. (2008) and more recently with Maciejewski et al. (2010), and determine a suggested rotational period of approximately 6 days for Kepler-6 (Figure 2). These specific cases are discussed below.

WASP-10. Currently, the radii of WASP-10b and its K5 host star are under debate. Christian et al. (2008) and Dittman et al. (2009) both find the radius of WASP-10b to be $1.28 R_{J}$, but Johnson et al. (2009) found a radius of $1.08 R_{J}$. This variation is explained by Johnson to be a difference in the transit depths of the normalized light curves, while Dittmann relates it to systematic errors from Johnson's optical system. Intervals of approximately one year interceded the data collection for these measurements and the Johnson and Dittmann teams only used data from a single transit for their findings. A contributing factor to the discrepancy could be star spots located off the transit chord causing a diminished transit depth in the light curve (Silva-Valio (2008); Valio; Boise 2010, these proceedings). If the data collected by Johnson et al. occurred during a period of increased stellar activity, this could cause a decreased transit depth. Although the required covering fraction is large, starspots have been found to cover up to $40 \%$ of the stellar disc (O'Neal et al. 1998).

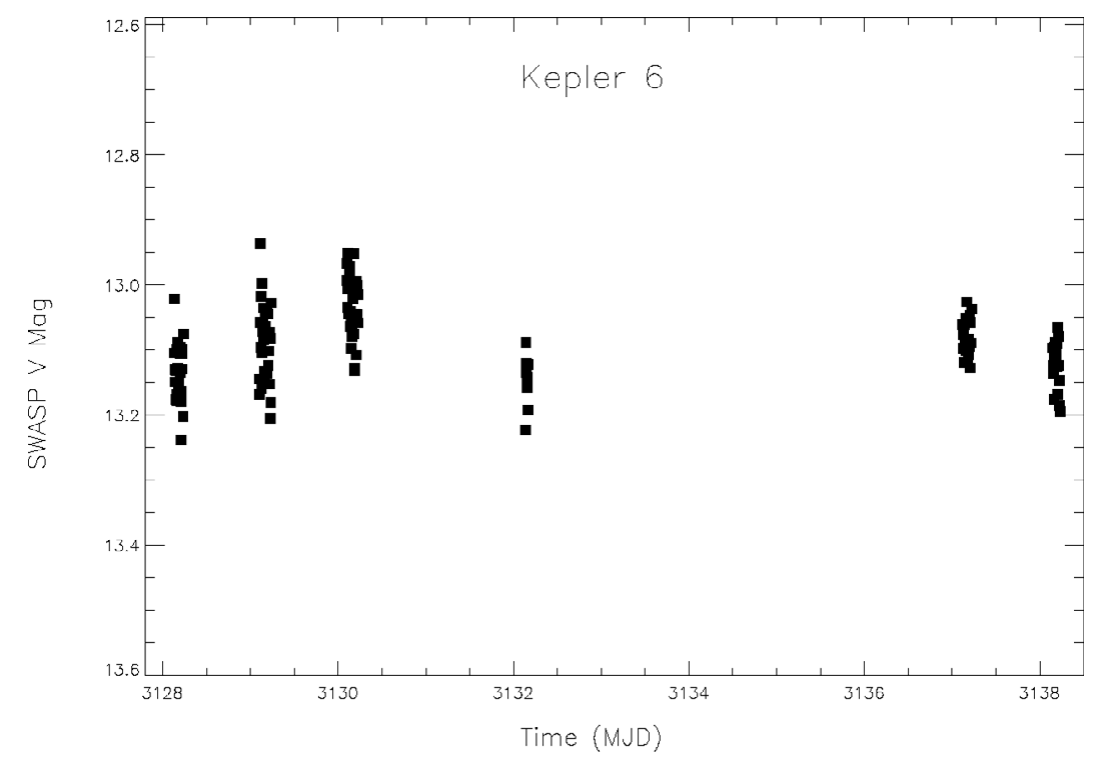

Figure 2. SuperWASP light curve of Kepler-6 from May 2004 (MJD 53128.5 corresponds to 03 May 2004). 
Kepler. Data on the Kepler targets have only recently been acquired by the Kepler mission. However, a review of the SuperWASP light curves for the Kepler targets has shown variation in some of the light curves and a rotational period of approximately 6 days for Kepler-6.

\section{Future}

We will continue to gather and analyze data on our current targets to establish activity cycles and rotational periods, and then investigate individual transits to derive both planetary and stellar parameters. Further interrogations of the SuperWASP and Kepler archives are under way and new targets being considered.

\section{Acknowledgements}

We thank the SuperWASP and Kepler projects for data access. We would also like to thank the CSUN Physics and Astronomy Department and the IAU 273 LOC for support of this project.

\section{References}

Bakos, G. À.; Torres, G.; Pàl, A.; Hartman, J.; Kovàcs, Gza; Noyes, R. W.; Latham, D. W.; Sasselov, D. D.; Sip:ocz, B.; Esquerdo, G. A.; and 14 coauthors 2010, Astrophys. J., 710, 1724

Basri, G., Walkowicz, L., Batalha, N. and 10 coauthors 2010, Astron. J., 141, 20

Charbonneau, D., Brown, T., \& Latham, D. 2000, Astrophys. J., 529, L45

Christian, D. J. \& Gibson, N. J. 2008, Mon. Not. R. Astron. Soc., 392, 1585

Dittman, J. A. \& Close, L. M. 2009, Astrophys. J., 701, 756

Dittman, J. A. \& Close, L. M. 2010, Astrophys. J., 717, 235

Fares, R. \& Donati, J. F. 2010, Mon. Not. R. Astron. Soc., 406, 409

Johnson, J., Winn, J., Cabrera, N. 2009. Astrophys. J., 692, 100

Maciejewski, G., Dimitrov, D., Neuhäuser, R. 2010. Mon. Not. R. Astron. Soc. In Press (arXiv:1009.4567v1)

O'Neal, D., Saar, S., Neff, J. 1998. Astrophys. J., 501, L73

Pollacco, D. L. \& Skillen, I. et al. 2006, Pub. Astron. Soc. Paci., 118, 1407

Rabus, M. \& Alonso, R. 2009, Astron. Astrophys, 494, 391

Schneider, J. 2000, ASPCS, 212, 284

Schneider, J. 2010, The Extrasolar Planets Encyclopedia, retrieved from www.exoplanet. eu/catalog-transit.php

Silva-Valio, A. 2008, Astrophys. J., 683, 179

Silva-Valio, A., Lanza, A. F., Alonso, R., Barge, P. 2010 Astron. Astrophys, 510, 25 